\title{
MISALIGNED DISKS IN THE BINARY PROTOSTAR IRS 43
}

Christian Brinch ${ }^{1}$, Jes K. Jørgensen ${ }^{2}$, Michiel R. Hogerheijde ${ }^{3}$, Richard P. Nelson ${ }^{4}$, and Oliver Gressel ${ }^{1}$

${ }^{1}$ Niels Bohr International Academy, The Niels Bohr Institute, University of Copenhagen, Blegdamsvej 17, DK-2100 Copenhagen $\varnothing$, Denmark; brinch@nbi.ku.dk ${ }^{2}$ Centre for Star and Planet Formation, The Niels Bohr Institute \& Natural History Museum of Denmark, University of Copenhagen, Øster Voldgade 5-7, DK-1350 Copenhagen K, Denmark

${ }^{3}$ Leiden Observatory, Leiden University, P.O. Box 9513, 2300 RA Leiden, The Netherlands

4 Astronomy Unit, Queen Mary University of London, Mile End Road, London E1 4NS, UK

Received 2016 August 12; revised 2016 September 6; accepted 2016 September 6; published 2016 October 11

\begin{abstract}
Recent high angular resolution $(\sim 0$ !' 2$)$ ALMA observations of the $1.1 \mathrm{~mm}$ continuum and of $\mathrm{HCO}^{+} J=3-2$ and HCN $J=3-2$ gas toward the binary protostar IRS 43 reveal multiple Keplerian disks that are significantly misaligned $\left(>60^{\circ}\right)$, both in inclination and position angle and also with respect to the binary orbital plane. Each stellar component has an associated circumstellar disk while the binary is surrounded by a circumbinary disk. Together with archival VLA measurements of the stellar positions over 25 years, and assuming a circular orbit, we use our continuum measurements to determine the binary separation, $a=74 \pm 4$ au, and its inclination, $i<30^{\circ}$. The misalignment in this system suggests that turbulence has likely played a major role in the formation of IRS 43 .
\end{abstract}

Key words: astrometry - protoplanetary disks - stars: formation

\section{INTRODUCTION}

Planets seem to be a common phenomenon with an estimated occurrence rate of at least one planet per star in the Milky Way (Cassan et al. 2012). Even binary stars are found to have planets orbiting, and we currently know about almost 100 exoplanets orbiting binaries, out of which about 10 are circumbinary. A common feature of all these planets around binary stars is that their orbits are well aligned with the orbit of the binary. The largest known deviation from co-planarity is 2.5 (Kostov et al. 2014). This alignment suggests that the stars condensed out of a single disk by fragmentation followed by planet formation in the same circumbinary disk through core accretion, a formation scenario which is supported by recent observations (Tobin et al. 2013, 2016). However, there is a strong selection effect because well-aligned systems are much easier to detect via transits. Theoretically, Artymowicz \& Lubow (1994) and Bate \& Bonnell (1997) showed that for close binaries with separation less than $100 \mathrm{au}$, circumstellar disks will form within the central cavity of the circumbinary disk. Such multiple protostellar systems have recently been found, e.g., GG Tau (Dutrey et al. 2014) and L1551 IRS 5 (Lim et al. 2016), both of which have circumstellar disks as well as a circumbinary disk that are well aligned. Jensen \& Akeson (2014) reported misaligned circumstellar disks in the binary star HK Tau, but no circumbinary disk is present in that system. In this Letter, we report observations of a binary protostar, IRS 43, which also has three separate disks, two circumstellar and one circumbinary, but these are all substantially misaligned $\left(\gg 5^{\circ}\right)$. IRS 43 (IRAS 16244-2434) is a binary protostellar system in the Rho Ophiuchus star-forming region $(d=120 \mathrm{pc}$; Knude \& $\mathrm{H} \emptyset \mathrm{g}$ 1998). It is described in the literature as a Class I object due to the shape of the spectral energy distribution (Wilking et al. 1989) and hence is assumed to be of the order of 100,000 years old. IRS 43 was discovered to be a binary by Girart et al. (2000) based on $3.6 \mathrm{~cm}$ continuum observations from the Very Large Array (VLA) taken in 1989. Following these authors, we will henceforth refer to the two stellar components as VLA 1 and VLA 2, with VLA 1 being the northernmost source and also the stronger of the two at millimeter and centimeter wavelengths. The binary was later confirmed by observations in the mid-infrared where, curiously enough, VLA 2 is seen to be the stronger source (Haisch et al. 2002; Duchêne et al. 2007).

\section{OBSERVATIONS AND DATA REDUCTION}

IRS 43 was observed by the Atacama Large Millimeter/ submillimeter Array (ALMA) on 2015 August 28, spending approximately $1 \mathrm{hr}$ on the source. Thirty-six antennas were in use on that night providing baselines from 15 to $1465 \mathrm{~m}$. Two phase calibrators, J1625-2527 and J1627-2426, were observed approximately every 7 minutes. The precipitable water vapor level was around $2 \mathrm{~mm}$ at the time of observing. We used Band 6 in our observations, and receivers were tuned to $267 \mathrm{GHz}$ $(1.1 \mathrm{~mm})$. Of the four spectral windows, one was set up to measure the continuum at $252 \mathrm{GHz}$ using the full $2 \mathrm{GHz}$ bandwidth. The remaining three spectral windows were used to measure the lines $\mathrm{HCO}^{+} J=3-2$ at $267.6 \mathrm{GHz}, \mathrm{HCN} J=3-2$ at $265.9 \mathrm{GHz}$, and $\mathrm{HC}^{18} \mathrm{O}^{+} J=3-2$ at $255.5 \mathrm{GHz}$. The latter was not detected. $\mathrm{HCO}^{+}$was measured at a spectral resolution of $61 \mathrm{kHz}$ corresponding to $68 \mathrm{~ms}^{-1}$ while HCN was observed at a slightly lower spectral resolution, $122 \mathrm{kHz}$ corresponding to $138 \mathrm{~ms}^{-1}$. Careful recalibration of the data did not improve the quality significantly compared to the delivered version calibrated by ALMA staff, so we settled on using the original version as provided by ALMA. We did experiment with selfcalibration of the continuum, but the improvement in signal-tonoise was negligible and did not reveal additional structure, so in the end we decided not to self-calibrate.

In this Letter, we also make use of archival data from the VLA as well as data published by Girart et al. (2000) and Curiel et al. (2003). Two additional data sets on IRS 43 were found in the NRAO VLA archive, project numbers AC0788 and AF0443, taken in 2005 and 2007 respectively. These two data sets were phase calibrated using CASA and astrometric positions of the protostellar components measured and added to the list of VLA astrometry. In addition, we utilize continuum data from ALMA observations of IRS 43 0." $4 \times 0$ ". 3 angular resolution from a larger survey of Class I protostars in 

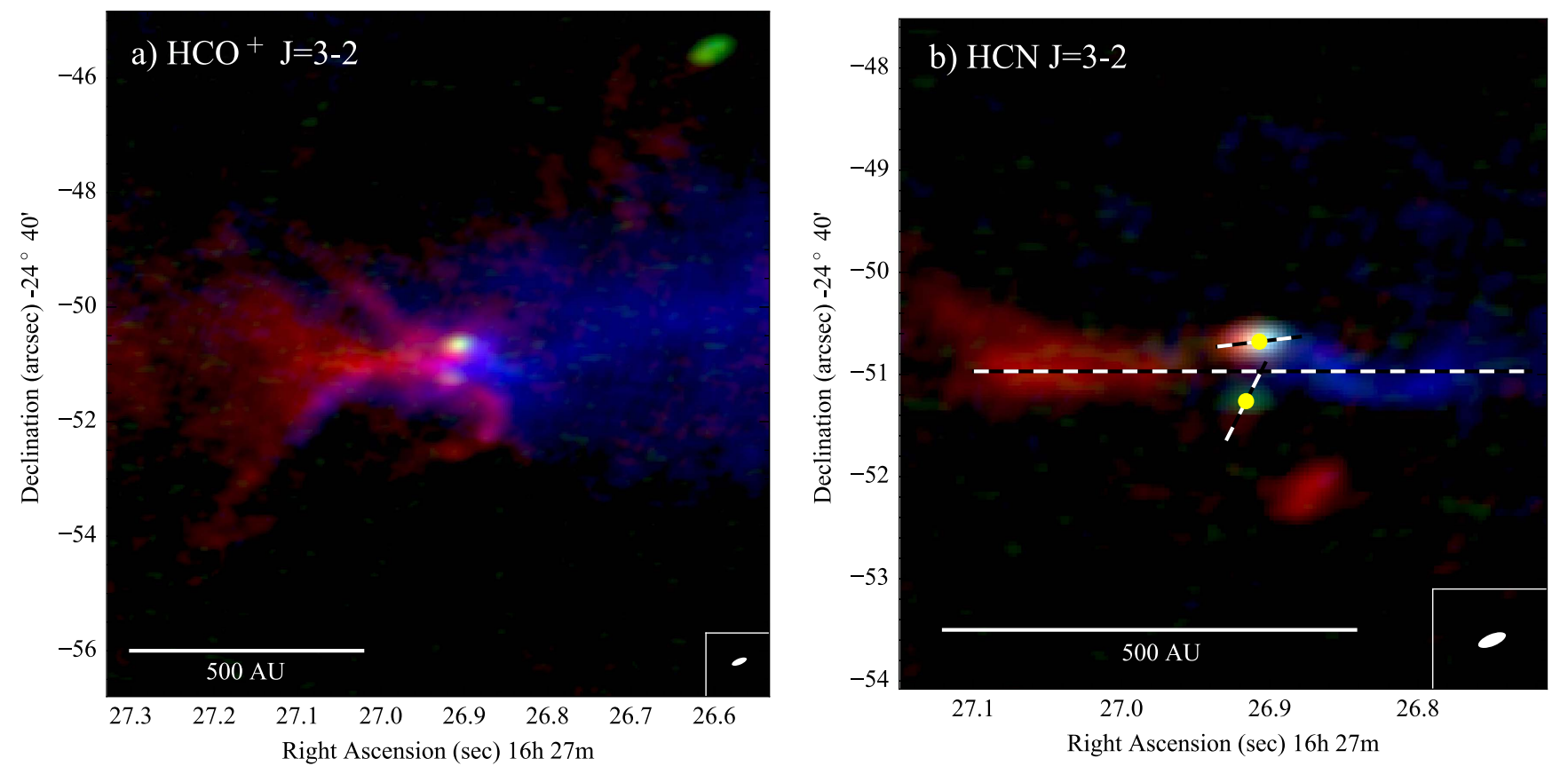

Figure 1. Three-color images of the continuum and line emission from IRS 43 . In both panels, green shows the $1.1 \mathrm{~mm}$ continuum, red is the integrated redshifted line emission, and blue is the blueshifted line emission. Panel (a) shows the $\mathrm{HCO}^{+} J=3-2$ emission, and panel (b) shows the HCN $J=3-2$ line. The intensity of all three colors has been normalized by the peak intensity. The white dashed lines in the right panel show the major axes of the three disk components. The positions of the two protostars are marked by yellow circles.

Ophiuchus (2013.1.00955.S, PI: Jes Jørgensen; E. Artur et al. 2016 , in preparation).

\section{RESULTS}

\subsection{Dust}

The continuum emission as observed by ALMA shows two distinct peaks, both of which are marginally resolved. The continuum is very compact with no trace of extended emission on scales larger than 0 "! 3 . The continuum is shown in green color in Figure 1, restored with a 0 !' $23 \times 0$ !" 15 beam. A third and very strong continuum source can also be seen in Figure 1 some 800 au to the northeast of VLA 1 and VLA 2. This source is known as GY263 and may or may not be gravitationally bound to IRS 43 .

The continuum emission originates from cold dust surrounding the the two protostars. If we assume a constant dust temperature of $15 \mathrm{~K}$ and a dust opacity $\kappa$ at $1.1 \mathrm{~mm}$ of $2.7 \mathrm{~cm}^{2} \mathrm{~g}^{-1}$, we can estimate the dust mass around the stars using

$$
M_{\text {dust }}=\frac{F_{\lambda} d^{2}}{\kappa_{\lambda} B_{\lambda}\left(T_{\text {dust }}\right)},
$$

where $d$ is the distance to the source and $B_{\lambda}(T)$ is the Planck function for temperature $T$. The continuum flux of VLA 1 and VLA 2 is $18.5 \mathrm{mJy}$ and $1.9 \mathrm{mJy}$, respectively, which gives total gas and millimeter dust masses of $770 M_{\oplus}$ and $20 M_{\oplus}$ after multiplying $M_{\text {dust }}$ with the canonical gas-to-dust ratio of 100 .

The fact that the continuum is only marginally resolved allows us to place upper limits to the size of the dust distributions. Given a beam size of $0 . " 3$, the dust extends no more than 20 au from the stars. The dust around VLA 1 may extend slightly further than that around VLA 2, but not more that $10 \%-20 \%$.

\subsection{Gas}

The $\mathrm{HCO}^{+} J=3-2$ and $\mathrm{HCN} J=3-2$ emission is shown in Figure 1 along with the continuum emission. Both lines are strong and clear. In these two figures, the integrated redshifted and the blueshifted emission is colored in red and blue, respectively. $\mathrm{HCO}^{+}$is seen to be a lot more extended than $\mathrm{HCN}$, particularly in the direction along the declination, but both tracers show a clear circumbinary red/blue asymmetry. $\mathrm{HCO}^{+}$shows an $\mathrm{X}$-shaped and slightly curved structure in both red and blue, which is not seen in $\mathrm{HCN}$ at all. This structure emits at large velocity offset only $\left(>7 \mathrm{kms}^{-1}\right)$ and is likely associated with an outflow or disk wind. The three disks are easily visible in HCN. In Figure 1, their axes are marked by white dashed lines. We can confirm that these structures are in fact Keplerian disks by looking at the velocity field as a function of position offsets. Figure 2 shows the PV-diagrams along the axes shown in Figure 1 for the circumbinary disk and the circumstellar disk around VLA 1. The signal is not strong enough to prove that the velocity gradient across VLA 2 is Keplerian, but Herczeg et al. (2011) found a disk in CO associated with VLA 2. We therefore assume that the velocity gradient seen in HCN across VLA 2 is due to this disk, and we adopt a position angle that lies along this gradient. In both PVdiagrams, a linear segment of emission at low velocities connects the red and the blue side across the center. This is expected for a Keplerian disk of finite radius. The origin of this emission is well explained by Lindberg et al. (2014). The blueshifted side of the circumbinary disk in $\mathrm{HCN}$ has an apparent warp. 

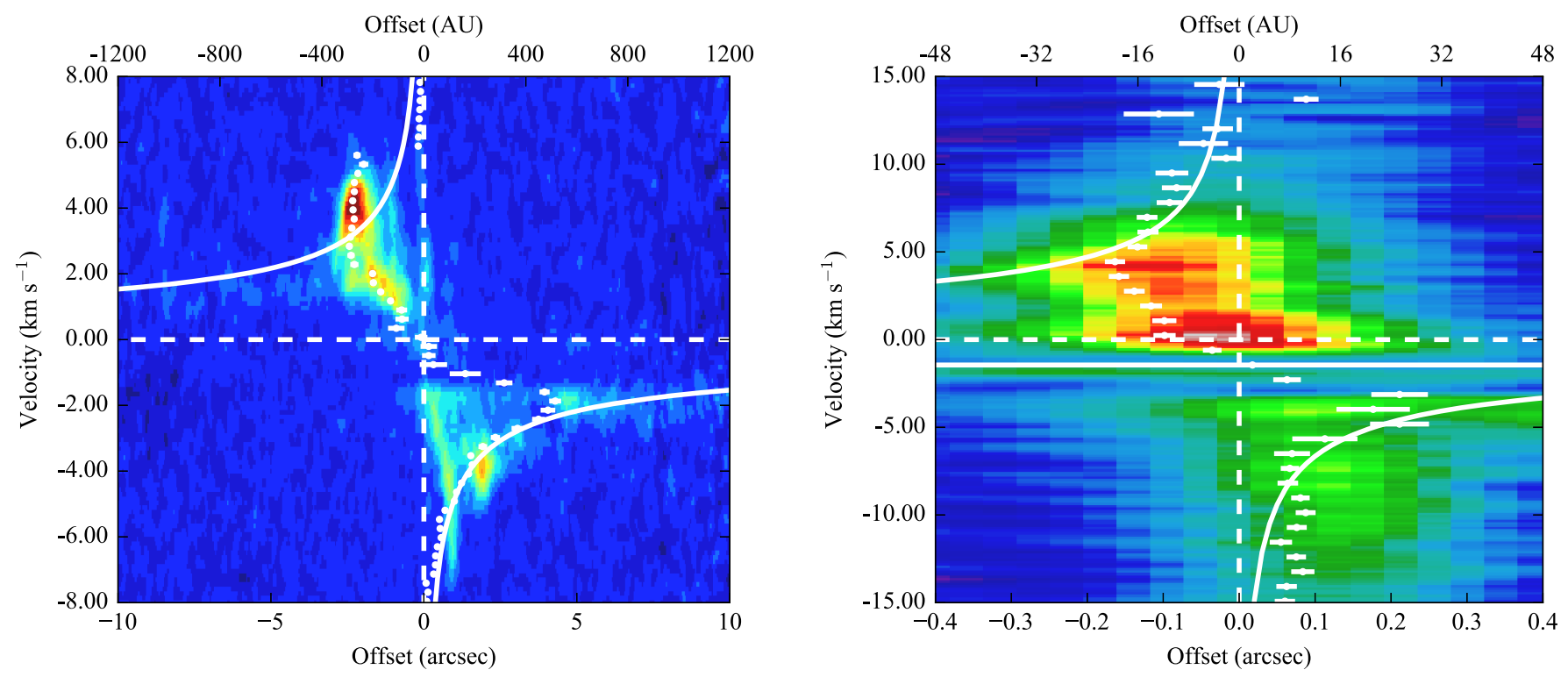

Figure 2. PV-diagrams of the HCN $J=3-2$ emission. The left panel shows the PV-diagram along a cut through the center of mass along the circumbinary disk midplane, and the right panel shows the PV-diagram along the major axis of the VLA 1 disk exactly through VLA 1 itself. The white markers show the peak position and error bar of a Gaussian fit to the emission in each channel. The full white lines show a Keplerian fit that is discussed in Section 4.
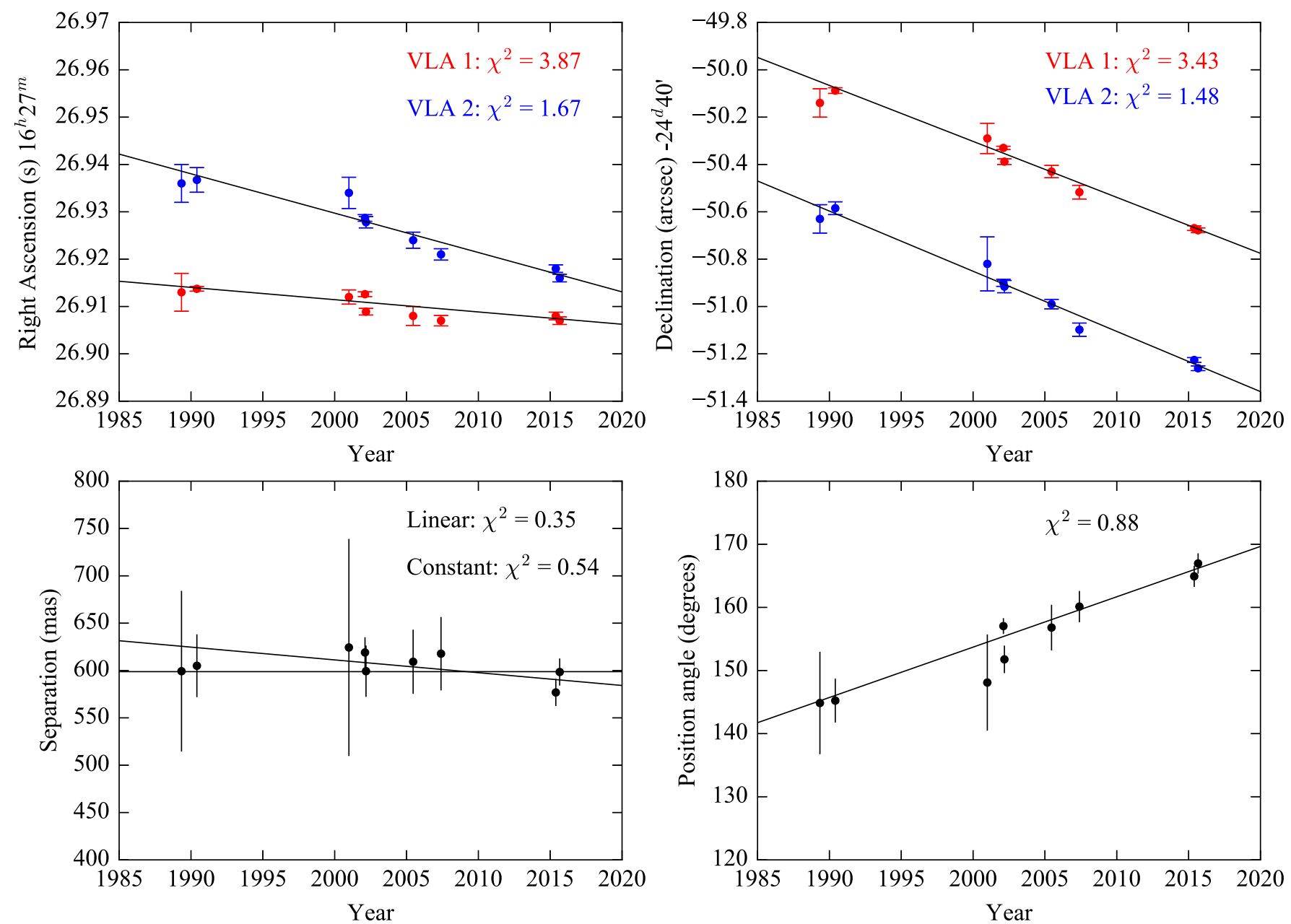

Figure 3. Measured positions of VLA 1 and VLA 2 over the span of 25 years (top panels) as well as the derived quantities of separation and position angle (bottom panels), also as a function of time. The figure shows the least squares fits to the data, and reduced $\chi^{2}$ values are given for each fit. 
Table 1

Best-fit Parameters

\begin{tabular}{lccc}
\hline \hline & \multicolumn{2}{c}{ Least Squares } & PIKAIA \\
\cline { 2 - 3 } Parameter & VLA 1 & VLA2 & CoM \\
\hline$\mu_{\text {R.A. }}\left(\right.$ mas yr $\left.^{-1}\right)$ & $-3.9(0.9)$ & $-12.5(1.0)$ & $-7.6(0.5)$ \\
$\mu_{\text {Decl. }}\left(\right.$ mas yr $\left.^{-1}\right)$ & $-23.6(0.9)$ & $-25.4(0.9)$ & $-25.3(0.4)$ \\
Separation $(m a s)$ & \multicolumn{2}{c}{$599(6)$} & $615(37)$ \\
Separation $(\mathrm{au})$ & \multicolumn{2}{c}{$72(1) \mathrm{au}^{\mathrm{a}}$} & $74(4) \mathrm{au}^{\mathrm{a}}$ \\
Period (years) & \multicolumn{2}{c}{$451.2(46.9)$} & $444.2(33.4)$ \\
Stellar mass ratio & \multicolumn{2}{c}{$\ldots$} & $0.500(0.018)$ \\
Total mass $\left(M_{\odot}\right)$ & \multicolumn{2}{c}{$1.80(0.42)$} & $2.01(0.47)$ \\
\hline
\end{tabular}

Note.

${ }^{\mathrm{a}}$ Assuming a distance of $120 \mathrm{pc}$.

\subsection{Astrometry}

Curiel et al. (2003) published a study in which they had followed the proper motion of IRS 43 using the VLA over a 12 year period, from 1990 to 2002 . They produced a lower limit to the total mass $\left(1.7 M_{\odot}\right)$ and an upper limit on the period (360 year) as well as an estimate of the binary separation and absolute proper motion of the system.

With our recent ALMA data and additional archival VLA data, we can add 13 years to the fraction of measured orbital period, which allow us to produce a more accurate determination of the orbital parameters including reliable error bars. The two derived quantities, separation and position, are shown in Figure 3. Least squares fits to the data are shown as well together with the reduced $\chi^{2}$ values of each fit. These values show that the fits are very robust. In the case of the binary separation, we have fitted both a linear function, allowing for a change of separation in time, as well as a constant, which would assume a circular orbit in the plane of the sky, i.e., constant separation. Based on the reduced $\chi^{2}$-values of the two fits, we cannot determine whether the separation of the protostars is constant in time or not, but within the error bars the data are certainly consistent with a circular orbit lying in, or very close to, the plane of the sky. If we allow for a non-zero inclination, we find that the data are inconsistent with an inclination above $30^{\circ}$ from the plane of the sky. The least squares fit parameters are given in Table 1 where we give the slope of the best-fitting line to the position angle data as the angular velocity.

In order to determine the orbit of the binary, we fit, for each of the two stars, a function of the form

$$
\begin{aligned}
\boldsymbol{r}(t)= & \boldsymbol{r}_{\mathrm{CoM} ; 0}+\boldsymbol{v}_{\mathrm{CoM}} t \\
& +d f\left[\cos \left(\omega t+\phi_{0}\right) \hat{\boldsymbol{x}}+\sin \left(\omega t+\phi_{0}\right) \hat{\boldsymbol{y}}\right],
\end{aligned}
$$

where $\boldsymbol{r}_{\mathrm{CoM} ; 0}$ is the position of the center of mass at $t_{0}, \boldsymbol{v}_{\mathrm{CoM}}$ is the velocity of the center of mass in the plane of the sky, $d$ is the projected separation of the stars, $f$ is the mass ratio of the stars, $\omega$ is the orbital frequency, and $\phi_{0}$ is the phase (i.e., position angle) at $t_{0}$. A total of six free parameters as the position of the center of mass is fixed by the positions of the stars together with the parameter $f$. The fitting is done by a genetic algorithm PIKAIA (Charbonneau 1995). We ran 20,000 optimization runs with different random number generator seeds and each run would return a slightly different optimal solution. We then fitted Gaussians to the distributions

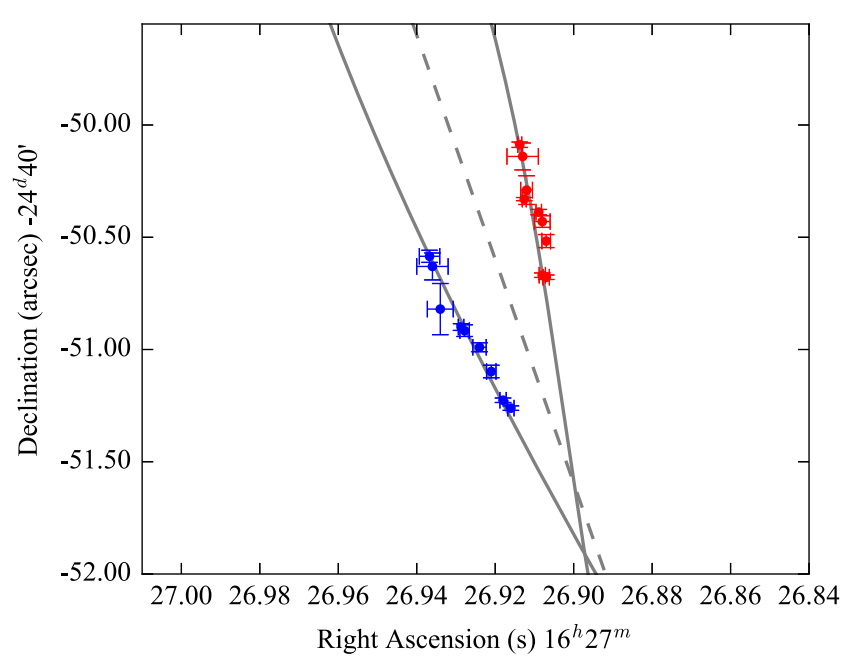

Figure 4. Measured positions of VLA 1 (red) and VLA 2 (blue). The gray lines show the best-fit trajectories, while the broken line shows the trajectory of the center of mass.

of output parameter values and taking the mean of these Gaussian fits as the most probable parameter value. These values are shown in Table 1 along with the values of the least squares fit. The best fit provided by PIKAIA is perfectly consistent with the values found by least squares fitting, but we also get a value for the additional mass ratio parameter, which we cannot obtain directly from the data. Surprisingly, the most probable value is $1 / 2$, which means that the two stars carry the exact same mass. The total stellar mass that we obtain here, purely from the determination of the apparent orbital period, is very consistent with the mass of $1.9 M_{\odot}$ obtained by Brinch \& Jørgensen (2013) based entirely on radiative transfer modeling of the velocity field in the circumbinary gas.

The trajectories of the two protostellar components and their measured positions on the sky are shown in Figure 4. The dashed line shows the trajectory of the center of mass.

\section{DISCUSSION AND CONCLUSIONS}

The most curious misalignment in the IRS 43 system is that of the circumbinary disk and the orbit of the binary itself. The orbital plane of the binary is strongly constrained by the constant separation of VLA 1 and VLA 2 in time to be close to the plane of the sky $\left(i<30^{\circ}\right)$, whereas the circumbinary disk is seen nearly edge-on. Not only does the aspect ratio of the circumbinary emission distribution prevent a line of sight inclination less than $\sim 60^{\circ}$, but the PV-diagram (Figure 2, left) is also very well described by a Keplerian profile using a mass of $1.8 M_{\odot}$ and an inclination of $70^{\circ}$. This inclination is consistent with the inclination found by Brinch \& Jørgensen (2013). If we use the slightly higher total mass of $2.01 M_{\odot}$ found by the PIKAIA fit, we need an inclination of $60^{\circ}$ to fit the PV-diagram. The red side of the HCN line has a strong nonKeplerian peak at a radius of $300 \mathrm{au}$. This peak masks the Keplerian signature on the red side at this radius, but the emission actually follows the Kepler profile, although at a very low signal to noise, out to about 600 au or slightly more. It is not clear what causes the bright spot on the red side at $300 \mathrm{au}$.

Similarly, we can fit the PV-diagram of the circumstellar disk around VLA 1 using a mass of $0.9 M_{\odot}$ and an inclination 
of $33^{\circ}\left(30^{\circ}\right.$, if we use $\left.1.005 M_{\odot}\right)$. We cannot determine the line of sight inclination of the circumstellar around VLA 2 due to low signal to noise, but this disk has a velocity gradient that is offset by almost $90^{\circ}$ with respect to the other two disks. The orientation of the disk around VLA 2 is further supported by the linear structure in $\mathrm{HCO}^{+}$, most likely an outflow, emerging from VLA 2 toward north-west and almost perpendicular to the velocity gradient.

The warp in the disk, most apparent in the blueshifted HCN emission, is expected from simulations (Larwood \& Papaloizou 1997), and it is also to be expected that a cavity in the circumbinary disk be carved by the binary (Artymowicz \& Lubow 1994). Whether such a cavity can be seen in our data is not clear, but there is a hint of a drop in $\mathrm{HCN}$ intensity in the central parts of the circumbinary disk. Higher-resolution observations by ALMA could reveal this.

The significant misalignment of this system suggests a formation scenario either driven by turbulent fragmentation (Padoan \& Nordlund 2002) or that the system has recently been perturbed, e.g., by ejection of a third stellar component. Another possibility is that the misalignment between the central binary and the circumbinary disk may simply be a signature of formation of the binary in a cloud, with infall of material with misaligned angular momentum, occurring late in the formation of the circumbinary disk. This would imply that the material from which the system formed was not in solid body rotation as is often considered the initial condition for star formation, but rather that the formation was triggered in a cloud with significant turbulence or substructure.

The question is whether IRS 43 will eventually align and form a planetary system that is similar to all the well-aligned systems found by Kepler. Bate et al. (2010) showed that, in particular for light disks, a misalignment of the rotation axis of a protostar and its disk may survive for the entire life time of the disk. In the case of IRS 43, alignment of disks with binaries tends to happen on the viscous timescale (Papaloizou \& Terquem 1995). For the two circumstellar disks, at the radius of 25 au, we estimate the viscous timescale to be between $8 \times 10^{5}$ and $8 \times 10^{6}$ years for values of the $\alpha$-viscosity parameter (Shakura \& Sunyaev 1973) between $10^{-2}$ and $10^{-3}$. This system could take a very long time to align $\left(\sim 10^{6}\right.$ years $)$, possibly beyond the lifetimes of the disks and certainly longer than the the present age of IRS $43\left(\sim 10^{5}\right.$ years; Brinch \& Jørgensen 2013).

\section{REFERENCES}

Artymowicz, P., \& Lubow, S. H. 1994, ApJ, 421, 651

Bate, M. R., \& Bonnell, I. A. 1997, MNRAS, 285, 33

Bate, M. R., Lodato, G., \& Pringle, J. E. 2010, MNRAS, 401, 1505

Brinch, C., \& Jørgensen, J. K. 2013, A\&A, 559, A82

Cassan, A., Kubas, D., Beaulieu, J.-P., et al. 2012, Natur, 481, 167

Charbonneau, P. 1995, ApJS, 101, 309

Curiel, S., Girart, J. M., Rodríguez, L. F., \& Cantó, J. 2003, ApJL, 582, L109

Duchêne, G., Bontemps, S., Bouvier, J., et al. 2007, A\&A, 476, 229

Dutrey, A., di Folco, E., Guilloteau, S., et al. 2014, Natur, 514, 600

Girart, J. M., Rodríguez, L. F., \& Curiel, S. 2000, ApJL, 544, L153

Haisch, K. E., Jr., Barsony, M., Greene, T. P., \& Ressler, M. E. 2002, AJ, 124,2841

Herczeg, G. J., Brown, J. M., van Dishoeck, E. F., \& Pontoppidan, K. M. 2011, A\&A, 533, A112

Jensen, E. L. N., \& Akeson, R. 2014, Natur, 511, 567

Knude, J., \& Høg, E. 1998, A\&A, 338, 897

Kostov, V. B., McCullough, P. R., Carter, J. A., et al. 2014, ApJ, 784, 14

Larwood, J. D., \& Papaloizou, J. C. B. 1997, MNRAS, 285, 288

Lim, J., Yeung, P. K. H., Hanawa, T., et al. 2016, ApJ, 826, 153

Lindberg, J. E., Jørgensen, J. K., Brinch, C., et al. 2014, A\&A, 566, A74

Padoan, P., \& Nordlund, A. 2002, ApJ, 576, 870

Papaloizou, J. C. B., \& Terquem, C. 1995, MNRAS, 274, 987

Shakura, N. I., \& Sunyaev, R. A. 1973, A\&A, 24, 337

Tobin, J. J., Chandler, C. J., Wilner, D. J., et al. 2013, ApJ, 779, 93

Tobin, J. J., Looney, L. W., Li, Z.-Y., et al. 2016, ApJ, 818, 73

Wilking, B. A., Lada, C. J., \& Young, E. T. 1989, ApJ, 340, 823 